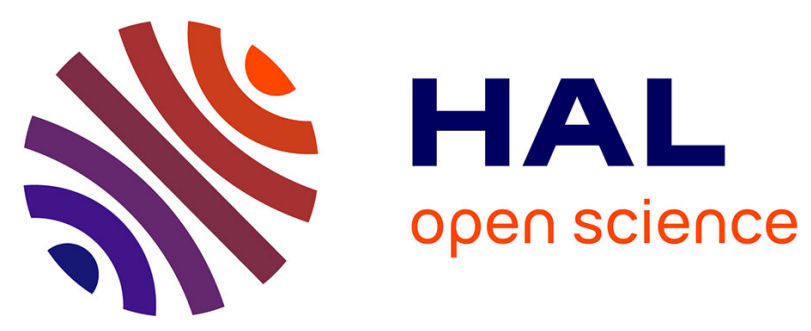

\title{
A High Speed Information-Centric Network in a Mobile Backhaul Setting
}

Diego Perino, Massimo Gallo, Roger Boislaigue, Leonardo Linguaglossa, Matteo Varvello, Giovanna Carofiglio, Luca Muscariello, Ben Houidi Zied

\section{- To cite this version:}

Diego Perino, Massimo Gallo, Roger Boislaigue, Leonardo Linguaglossa, Matteo Varvello, et al.. A High Speed Information-Centric Network in a Mobile Backhaul Setting. ACM Conference on Information-Centric Networking, Sep 2014, Paris, France. 10.1145/2660129.2660134. hal$01101463 \mathrm{v} 2$

\section{HAL Id: hal-01101463 \\ https://hal.inria.fr/hal-01101463v2}

Submitted on 27 Jan 2015

HAL is a multi-disciplinary open access archive for the deposit and dissemination of scientific research documents, whether they are published or not. The documents may come from teaching and research institutions in France or abroad, or from public or private research centers.
L'archive ouverte pluridisciplinaire HAL, est destinée au dépôt et à la diffusion de documents scientifiques de niveau recherche, publiés ou non, émanant des établissements d'enseignement et de recherche français ou étrangers, des laboratoires publics ou privés. 


\title{
A High Speed Information-Centric Network in a Mobile Backhaul Setting
}

\author{
Diego Perino*, Massimo Gallo*, Roger Boislaigue*, Leonardo Linguaglossa ${ }^{\ddagger}$ \\ Matteo Varvello*, Giovanna Carofiglio*, Luca Muscariello ${ }^{ \pm}$, Zied Ben Houidi* \\ * Bell Labs, Alcatel-Lucent, first.last@alcatel-lucent.com, $\ddagger$ INRIA, first.last@inria.com
}

\pm Orange, first.last@orange.com

\begin{abstract}
We demonstrate a high speed Information-Centric Network in a mobile backhaul setting. Specifically, we show the feasibility of an information aware data plane and we highlight the significant benefits it provides in terms of both user experience and network provider cost in the backhaul setting. Our setup consists of high-speed ICN devices employed in a down-scaled realistic representation of a mobile backhaul topology, fed with traffic workloads characterized from Orange's mobile network.
\end{abstract}

\section{Categories and Subject Descriptors}

C.2.1 [Network Architecture and Designs]: Network communications; C.2.6 [Internetworking]: Routers

\section{General Terms}

Design, Implementation, Experiments

\section{Keywords}

ICN, forwarding, router, architecture,protocols.

\section{INTRODUCTION}

Internet traffic and especially its mobile variant are rapidly increasing. As an example, mobile traffic is growing at the incredible rate of about $66 \%$ every year in the timeframe 2012-2017. On the one hand, this growth is driven by the radical terminal evolution; on the other hand, it is due to a shift of Internet usage from host-centric to informationcentric. The Internet is not used anymore as a medium to connect machines or hosts, but rather to connect people with content they are interested in.

Since such radical shift in Internet usage was not followed by an architectural change, inefficiencies quickly arose. Many of them are especially noticeable in the mobile network segment. They translate into difficulties in dynamic content-to-location mapping, mobility management, multicasting, multipath and multi-homing.

Permission to make digital or hard copies of part or all of this work for personal or classroom use is granted without fee provided that copies are not made or distributed for profit or commercial advantage and that copies bear this notice and the full citation on the first page. Copyrights for thirdparty components of this work must be honored. For all other uses, contact the Owner/Author.

Copyright is held by the owner/author(s).

ICN'14, September 24-26, 2014, Paris, France.

ACM 978-1-4503-3206-4/14/09.

http://dx.doi.org/10.1145/2660129.2660134.
Application layer solutions and bandwidth over provisioning have been so far the ways to mask such inefficiencies.

From a technical perspective, realizing efficient data delivery without capacity over-dimensioning demands for a flexible and adaptive traffic control coupled with in-network caching. However, due to the host centric nature of today's network architecture, there is a lack of mechanisms that natively perform dynamic and flexible information aware traffic control. This situation is even worse in mobile backhauls whereby, for mobility management reasons, the traffic is tunneled between backhaul ingress/egress nodes (i.e., eNodeB and Service Gateway).

We believe that Information-centric Networking (ICN) provides a natural answer to such needs by rethinking network data delivery around content/information. ICN introduces a connection-less name-based transport model, enables innetwork caching and multi-point to multi-point communication.

In this demonstration, we build a high-speed prototype of an information aware data plane spanning an entire network, including content consumers and content producers. To provide evidence of the ICN benefits in a realistic use case, our setting is based on a down-scaled mobile backhaul topology, and traffic workloads characterized from Orange's mobile network.

\section{INFORMATION AWARE DATA PLANE}

This section describes the information aware data plane we implemented for this demonstration. Specifically: i) we enrich the original ICN data plane defined in [2] with forwarding, traffic control and caching mechanisms described in the following sections; ii) we propose a design to realize an ICN data plane at high-speed. Our solution remains compatible with current Internet architecture and can be integrated in existing network equipment.

\subsection{Request Routing and Forwarding}

ICN nodes forward user requests by name and in a hopby-hop fashion towards a permanent copy of the requested content item. To this goal, every node has a name-based forwarding table, the FIB, that associates one or more potential next hops towards a set of content items. We developed a dynamic forwarding algorithm [1] that selects a next forwarding hop while achieving optimal throughput and minimum network cost. ICN nodes also keep track of received requests in order to return content chunks to the user following the reverse request path. This functionality is achieved via a pending request table, also called PIT. 


\subsection{Pull Based Connection-less Transport}

Data transfer is triggered by user requests addressed to chunks of the requested content item, i.e., pull based model. Rate and congestion control are performed at the end user by mean of a connection-less, yet stateful transport protocol. The receiver process maintains a pipeline of requests whose size is controlled by a window-based AIMD mechanism. For the demonstration, we implement the congestion control mechanism we proposed in [1] which realizes remote active queue management, based on the estimate of round trip delay per route. This protocol optimally allocates bandwidth resources among users in a fair and efficient fashion [1].

\subsection{In Network Caching}

In an ICN network, requests for the same data are served in network with no need to fetch any bytes from the original server/repository. Also, packet losses can be recovered in network, with no need for the sender to identify and retransmit the lost packet.

ICN's content-awareness enables a novel usage of buffers at the network nodes. In ICN, buffers are used to absorb input/output rate unbalance, as in today's IP networks, but also to cache in-transit data. Storage resources can also be added to a router augmenting its caching capabilities. We call content store, or CS, the data structure enabling caching at an ICN node.

\subsection{System Design}

To integrate ICN mechanisms within today's Internet architecture, we deploy ICN as an overlay over IP.

Several network devices currently employed in the Internet architecture are based on programmable elements, e.g., network processors. It is thus possible to integrate ICN mechanisms, name-based forwarding and caching, via simple firmware upgrades with no need to install ad-hoc hardware. We implement the required data structures, namely PIT, FIB and CS, and associated lookup algorithms following the design guidelines proposed in our previous work $[3,5,4]$. In addition, we implement the dynamic request forwarding scheme presented in [1].

End-host mechanisms like rate or congestion control can be implemented either at proxies co-located with network elements, or at the application layer directly at the end user. In this demonstration, we implement the multipath congestion-control algorithm described in [1] as an application over UDP/IP.

\section{DEMONSTRATION}

The demonstration platform consists of: i) a content router testbed, with a set of hardware nodes running the ICN data plane described above; ii) custom application layer data retrieval/server implementations running on general purpose servers.

For the demonstration, we realize the network topology shown in Figure 1, which is a representative down-scaled model of a mobile backhaul topology. To this end, we use hardware traffic shapers and L2 tunneling between physical boards. ICN content requests are generated by a generic data retrieval application that uses the end-host congestion controller described above. The workload we use for our demonstration follow a traffic profile characterized from Orange mobile network traces.

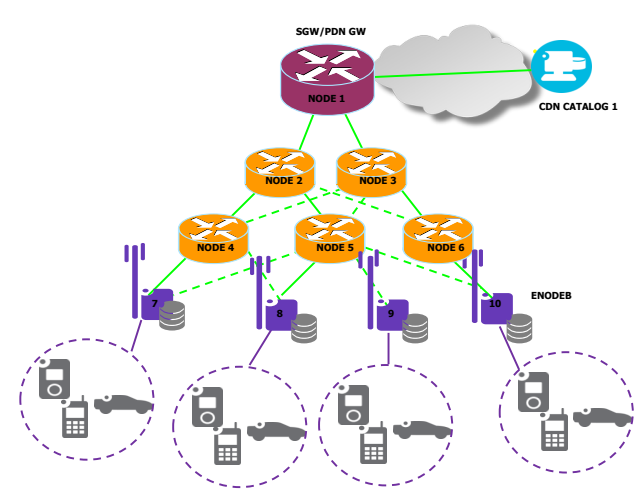

Figure 1: Down-scaled backhaul model topology used in the demonstration.

With the above described demonstration setup, we show the advantages of adopting an ICN data plane in this scenario, by enabling and disabling ICN functionalities. We compare our solution with the technology currently deployed in the mobile backhaul, characterized by IP tunnels between ingress/egress nodes (i.e., 3GPP standard), in terms of both user experience and network provider costs.

\section{CONCLUSION}

In this demonstration we have focused on a high speed Information-Centric Network in a mobile backhaul setting. The demonstration shows that the introduction of an ICN data plane is feasible and scalable Also ICN significantly reduces the content delivery time which improves end-user experience. Last but not least, ICN largely reduces the traffic load on the mobile backhaul with relevant bandwidth, and hence cost, savings.

\section{ACKNOWLEDGMENTS}

This work has been partially carried out in the framework of the common research laboratory between INRIA and Bell Labs, Alcatel-Lucent.

\section{REFERENCES}

[1] G. Carofiglio, M. Gallo, L. Muscariello, M. Papalini, and S. Wang. Optimal multipath congestion control and request forwarding in information-centric networks. In proc. of ICNP, 2013.

[2] V. Jacobson, D. K. Smetters, J. D. Thronton, M. F. Plass, N. H. Briggs, and R. L. Braynard. Network Named Content. In proc. of ACM CoNext, Rome, Italy, Dec. 2009.

[3] D. Perino and M. Varvello. A reality check for content centric networking. In proc. of ACM Sigcomm ICN workshop, Toronto, Canada, Aug. 2011.

[4] D. Perino, M. Varvello, L. Linguaglossa, R. Laufer, and R. Boislaigue. Caesar: A content router for high-speed forwarding on content names. In proc. of ACM/IEEE ANCS, Los Angeles, CA, USA, 2014.

[5] M. Varvello, D. Perino, and L. Linguaglossa. On the design and implementation of a wire-speed pending interest table. In proc. of IEEE Infocom NOMEN workshop, Turin, Italy, Aug. 2013. 